\title{
Validez del Pulmonary Embolism Rule-Out Criteria (PERC) para descartar embolia pulmonar en pacientes con bajo riesgo a gran altitud
}

\author{
Validity of the Pulmonary Embolism Rule-Out \\ Criteria (PERC) for ruling out pulmonary embolism \\ in low-risk patients at high altitudes
}

\author{
Alirio Rodrigo Bastidas-Goyes, Luis Felipe Reyes-Velasco, Estefan Ramos-Isaza, \\ Laura María Rodríguez-Jiménez, María Lygia Mondragón-Bravo, Juan Sebastián Monzón-Duarte, \\ Salvador Alejandro Scotti-D'Ovidio, Nathaly Vannesa Jiménez-Briceño, \\ María Fernanda Gómez-Rojas, Bobby Stefen Camacho-Palacios, \\ Natalia Moreno-Jaramillo, Juliana IzQuierdo-Polanco • Chía (Colombia)
}

DOI: https://doi.org/10.36104/amc.2021.2010

\section{Resumen}

Objetivos: validar el rendimiento diagnóstico del puntaje PERC para descartar embolia pulmonar en pacientes de bajo riesgo a gran altitud (>2500 $\mathrm{msnm})$.

Metodología: estudio de corte transversal con análisis de prueba diagnóstica en pacientes mayores de 18 años con sospecha diagnóstica de embolia pulmonar al ingreso o durante la hospitalización llevado a toma de angiotomografía de tórax desde agosto de 2009 hasta enero de 2020 en un centro de tercer nivel ubicado en la Sabana de Bogotá, se evaluó el rendimiento del puntaje PERC (Embolism Rule-Out Criteria) calculado con una $\mathrm{SatO} 2<95 \%$ y una $\mathrm{SatO} 2<90 \%$ en pacientes con diferentes niveles de riesgo según los puntajes de Wells, Ginebra y Pisa para embolia pulmonar.

Resultados: mil ochenta y siete ingresaron al ánalisis final, $42 \%$ con EP. Se encontró para los pacientes clasificados de bajo riesgo por el puntaje de Wells un ACOR para el PERC calculado con $\mathrm{SatO} 2<95 \%$ de 0.56 (IC-95\%:0.50-0.62) ( $\mathrm{p}=0.049$ ), y para el PERC calculado con $\mathrm{SatO} 2<90 \%$ de 0.60 (IC-95\%:0.54-0.66) ( $\mathrm{p}=0.002)$, el ACOR para sujetos clasificados de bajo riesgo por el puntaje de Ginebra con un PERC calculado con SatO2<95\% fue de: 0.53 (IC-95\%:0.45-0.60) ( $\mathrm{p}=0.459)$ y para un PERC calculado con SatO2<90\% fue de: 0.55 (IC-95\%:0.47-0.62) (P=0.218), el ACOR para sujetos clasificados con menos de $10 \%$ de probabilidad para EP por el puntaje de Pisa con un PERC calculado con SatO2<95\% fue de: 0.54 (IC-95\%:0.44-0.64)(p=0.422) y para un PERC calculado con SatO2<90\% fue de: 0.56 (IC-95\%:0.46-0.66) $(\mathrm{p}=0.236)$.

Conclusiones: el puntaje PERC calculado con una saturación de oxígeno $<90 \%$ tiene un rendimiento diagnóstico similar al puntaje PERC calculado con una saturación de oxígeno <95\% para descartar EP en pacientes clasificados en bajo riesgo con puntaje de Wells a gran altitud (>2500 msnm). (Acta Med Colomb 2021; 46. DOI: https://doi.org/10.36104/amc.2021.2010).

Palabras clave (DeCS): embolia pulmonar, reglas de decisión clínica, diagnóstico, probabilidad, altitud y reproducibilidad de los resultados.

\footnotetext{
Abstract

Objectives: to validate the diagnostic yield of the PERC score for ruling out pulmonary embolism in low-risk patients at high altitudes (>2500 meters above sea level [ASL]).

Methods: a cross-sectional study with diagnostic test analysis in patients over the age of 18 with suspected pulmonary embolism on admission or during hospitalization, who underwent chest computed tomography angiography between August 2009 and January 2020 in a tertiary care hospital located on the Bogotá savannah. The yield of the PERC score was assessed, calculated with an
}

Dr. Alirio Rodrigo Bastidas-Goyes: Internista, Neumólogo, Epidemiólogo. Profesor Asistente, Facultad de Medicina Universidad de La Sabana; Dr. Luis Felipe Reyes-Velasco: Intensivista, Doctor en Medicina Translacional. Profesor Asociado Facultad de Medicina Universidad de La Sabana; Dr. Estefan Ramos-Isaza: Médico General. Estudiante de Maestría en Epidemiología, Universidad de La Sabana; Dres. Laura María Rodríguez-Jiménez, María Lygia Mondragón-Bravo, Juan Sebastián Monzón-Duarte, Salvador Alejandro ScottiD’Ovidio, Nathaly Vannesa Jiménez-Briceño, María Fernanda Gómez-Rojas, Bobby Stefen Camacho Palacios, Natalia Moreno-Jaramillo, Juliana Izquierdo-Polanco: Médicos Generales Universidad de la Sabana. Chía (Colombia). Correspondencia: Dr. Dr. Alirio Rodrigo Bastidas-Goyes. Chía (Colombia). E-Mail: aliriorodrigo@yahoo.com Recibido: 10/IX/2020 Aceptado: 15/VI/2021 
$\mathrm{SaO} 2<95 \%$ and an $\mathrm{SaO} 2<90 \%$ in patients with different risk levels according to the Wells, Geneva and Pisa scores for pulmonary embolism.

Results: one thousand eighty-seven were included in the final analysis, $42 \%$ with PE. Patients classified as low-risk using the Wells score had a PERC ACOR calculated with $\mathrm{SaO} 2<95 \%$ of 0.56 (95\%CI:0.50-0.62) ( $\mathrm{p}=0.049)$, and calculated with $\mathrm{SaO} 2<90 \%$ of 0.60 (95\%CI:0.54-0.66) $(\mathrm{p}=0.002)$. The ACOR for subjects classified as low-risk using the Geneva score, with a PERC calculated with $\mathrm{SaO}<<5 \%$, was: 0.53 (95\%CI:0.45-0.60) $(\mathrm{p}=0.459)$ and for a PERC calculated with $\mathrm{SaO} 2<90 \%$ it was: 0.55 (95\%CI:0.47-0.62) $(\mathrm{P}=0.218)$. The ACOR for subjects with a less than $10 \%$ probability of $\mathrm{PE}$ according to the Pisa score classification, with a PERC calculated with $\mathrm{SaO} 2<95 \%$, was: 0.54 (95\%CI:0.44-0.64)(p=0.422), and for a PERC calculated with $\mathrm{SaO} 2<90 \%$ it was: $0.56(95 \% \mathrm{CI}: 0.46-0.66)(\mathrm{p}=0.236)$.

Conclusions: the PERC score calculated with an oxygen saturation $<90 \%$ has a similar diagnostic yield to the PERC score calculated with an oxygen saturation $<95 \%$ for ruling out PE in patients classified as low-risk by the Wells score at high altitudes (>2,500 meters ASL). (Acta Med Colomb 2021; 46. DOI: https://doi.org/10.36104/amc.2021.2010).

Keywords (DeCS): pulmonary embolism, clinical decision rules, diagnosis, probability, altitude and reproducibility of the results.

\section{Introducción}

La embolia pulmonar (EP) es una condición que afecta las arterias pulmonares y sus ramificaciones y que si no se sospecha y se diagnóstica a tiempo, puede resultar potencialmente mortal (1). Se caracteriza por un cuadro clínico inespecífico dado principalmente por disnea súbita, dolor torácico de tipo pleurítico (se exacerba con el esfuerzo inspiratorio), taquicardia, taquipnea, fiebre y tos seca o hemoptisis, con posibilidad de deterioro del estado general hasta presentar inestabilidad hemodinámica e incluso paro cardiorrespiratorio (2). Los síntomas respiratorios pueden ser similares o los encontrados en otras enfermedades como el síndrome coronario agudo, neumotórax y otras patologías pulmonares siendo necesario para su diagnóstico diferencial la realización de pruebas de laboratorio y angiotomografía computarizada (ATC) de las arterias pulmonares (3).

Dado que la evaluación de cada síntoma por separado para el diagnóstico de embolia pulmonar es inespecífica, se utiliza la suma de signos, síntomas y hallazgos de laboratorio para sospechar o descartar esta patología, en los últimos años se ha popularizado el uso de escalas de predicción clínica para guiar la conducta en la evaluación de estos pacientes. Entre ellas, una de las más difundidas es la escala de Wells que evalúa signos vitales de ingreso como la frecuencia cardíaca, el cuadro clínico sospechoso EP y antecedentes de importancia, y su resultado agrupa a los pacientes dependiendo el punto de corte que se utilice, así, los pacientes pueden ser clasificados con probabilidad alta, moderada y baja (tres niveles) o en probabilidad alta y baja (dos niveles) para EP (4-6). De forma similar otros puntajes como las escalas de Ginebra y Pisa que adicionalmente a los síntomas clínicos evalúan hallazgos paraclínicos, también han demostrado ser útiles en el abordaje diagnóstico de esta enfermedad $(7,8)$.

Estas escalas han sido ampliamente validadas en diferentes poblaciones y en nuestro medio para el diagnóstico de embolia pulmonar (9-11). Sin embargo, puntajes como el PERC el cual es utilizado para descartar está condición tienen información bibliográfica limitada en poblaciones especiales como las que se encuentran a gran altitud. El PERC que evalúa ocho variables clínicas incluido la saturación arterial de oxígeno (SatO2), ha demostrado su utilidad para descartar EP. Un puntaje de PERC de cero podría tener un rendimiento para excluir EP similar a un dímero D negativo $(12,13)$. No obstante, a gran altitud utilizar la SatO2 $<95 \%$ para construir el puntaje de PERC, puede influir en el rendimiento diagnóstico de este puntaje. Teniendo en cuenta que la saturación disminuye a medida que aumenta la altitud, es necesario evaluar si este puntaje requiere algún tipo de ajuste para su cálculo en poblaciones a gran altitud (mayor a $2500 \mathrm{msnm})(14,15)$. El objetivo de este estudio es valorar el rendimiento diagnóstico del puntaje PERC para descartar EP con diferentes valores de saturación de oxígeno en pacientes clasificados de bajo riesgo por las escalas de Wells, Ginebra y Pisa.

\section{Métodos}

Se llevó a cabo un estudio de corte transversal con análisis de prueba diagnóstica en sujetos con sospecha de embolia pulmonar, atendidos en los servicios de urgencias y hospitalización de la Clínica Universidad de la Sabana (Chía, Colombia), entre agosto de 2009 a enero de 2020. Se incluyeron todos los sujetos mayores de 18 años con realización de ATC de tórax por sospecha clínica de EP. Se excluyeron sujetos con realización del examen por sospecha de otras patologías como aneurisma de aorta, sospecha de trauma vascular, sospecha de patología aórtica no traumática, síndrome aórtico agudo, sujetos sin información o no localización de la historia clínica por problemas de sistema de información y sujetos con reporte de lectura radiología no disponible. Para la realización del presente estudio se 
cuenta con aprobación por parte del comité de ética de la Clínica Universidad de la Sabana.

Se registraron todas las variables clínicas y paraclínicas necesarias para la construcción de los puntajes de Wells, Ginebra revisado, Pisa con hallazgos radiográficos y PERC siguiendo la recomendación de los autores de los estudios originales para la construcción de cada uno de estos puntajes al momento de la sospecha diagnóstica de la $\operatorname{EP}(13,16,17)$. Se incluyeron de manera independiente los datos registrados en la anamnesis (variables demográficas, comorbilidades y sintomatología), hallazgos al examen físico, reportes de dímero D, radiografía de tórax, electrocardiograma, angiotomografía computarizada de arterias pulmonares, necesidad de ventilación mecánica, ingreso a unidad de cuidado intensivo y estado al egreso de la institución.

Cada uno de los puntajes se calculó de manera numérica y posteriormente se clasificó según su la probabilidad de EP; el puntaje de Wells en tres niveles (baja: $<2$, intermedia: 2-6 y alta: $>6)(6,18)$ y dos niveles (menos probable $\leq 4$ y probable $>4$ ) (19), el puntaje de Ginebra revisado en tres niveles (bajo: 0-3, intermedio: 4-10, alto 11-22) (20), el puntaje de Pisa en cuatro niveles (baja: 0-10, intermedio: 11-50, moderada alta: 51-90 y alta El 91-100) (21). El puntaje de PERC se calculó en dos niveles: probable $(\geq 1)$ o no probable $(=0)$ $(12,13)$. El diagnóstico de EP se realizó con el resultado de la ATC leída por radiólogo como estudio positivo para embolia pulmonar $(22,23)$. El tamaño de muestra se calculó de acuerdo con al estudio original de Kline et al, con el cual para una sensibilidad de $96 \%$, especificidad de $27 \%$, prevalencia de $8 \%$, nivel de precisión de $5 \%$ y un nivel de confianza de $95 \%$, se requerían un mínimo de 751 sujetos, los sujetos incluidos ingresaron de manera secuencial hasta completar el número requerido.
Los datos obtenidos de las historias clínicas fueron recolectados mediante el software de captura de datos electrónicos RedCap, para posteriormente ser analizados con el programa estadístico SPSS-25 licenciado. Se realizó una revisión inicial de los datos por variable para valorar el porcentaje de pérdida de datos. Posteriormente las variables cualitativas se resumieron en frecuencias y porcentajes y las cuantitativas según su distribución, si es normal en promedio y desviación estándar y si es no normal en mediana y rango intercuartílico, se realizó un análisis bivariado con cada una de las variables estudiadas y el diagnóstico de EP, las variables cuantitativas se compararon según su distribución con $t$ de student o U de Mann Whitney y las cualitativas con $c h i^{2}$. Se calculó un primer puntaje de PERC dando el valor de un punto a la $\mathrm{SatO} 2<95 \%$ y un segundo puntaje de PERC dando un punto a la $\mathrm{SatO} 2<90 \%$, el resultado de los dos puntajes de PERC agrupados por las diferentes categorías de riesgo de los puntajes Wells, Ginebra y Pisa se compararon con la positividad o negatividad de EP en la ATC, el valor de SatO2 $<90 \%$ fue escogido, teniendo en cuenta el estudio de González-García et al. de 2013 y 2020 acerca de los valores de gases arteriales a 2640 m.s.n.m (24). Posteriormente se estimó la sensibilidad, especificidad y área bajo la curva de características operativas del receptor (ACOR), con un intervalo de confianza de $95 \%$ para el puntaje PERC calculado con SatO2<95\% y del puntaje de PERC calculado con Sat $<90 \%$, se consideró una p estadísticamente significativa $<0.05$. Se siguieron las recomendaciones éticas de Helsinki y la resolución 8430 de 1993 para investigación en humanos, considerándose este estudio sin riesgo y que no requiere de la realización de consentimiento informado, así mismo, se siguieron las recomendaciones de la Ley de Habeas Data para el manejo y reserva de datos personales.

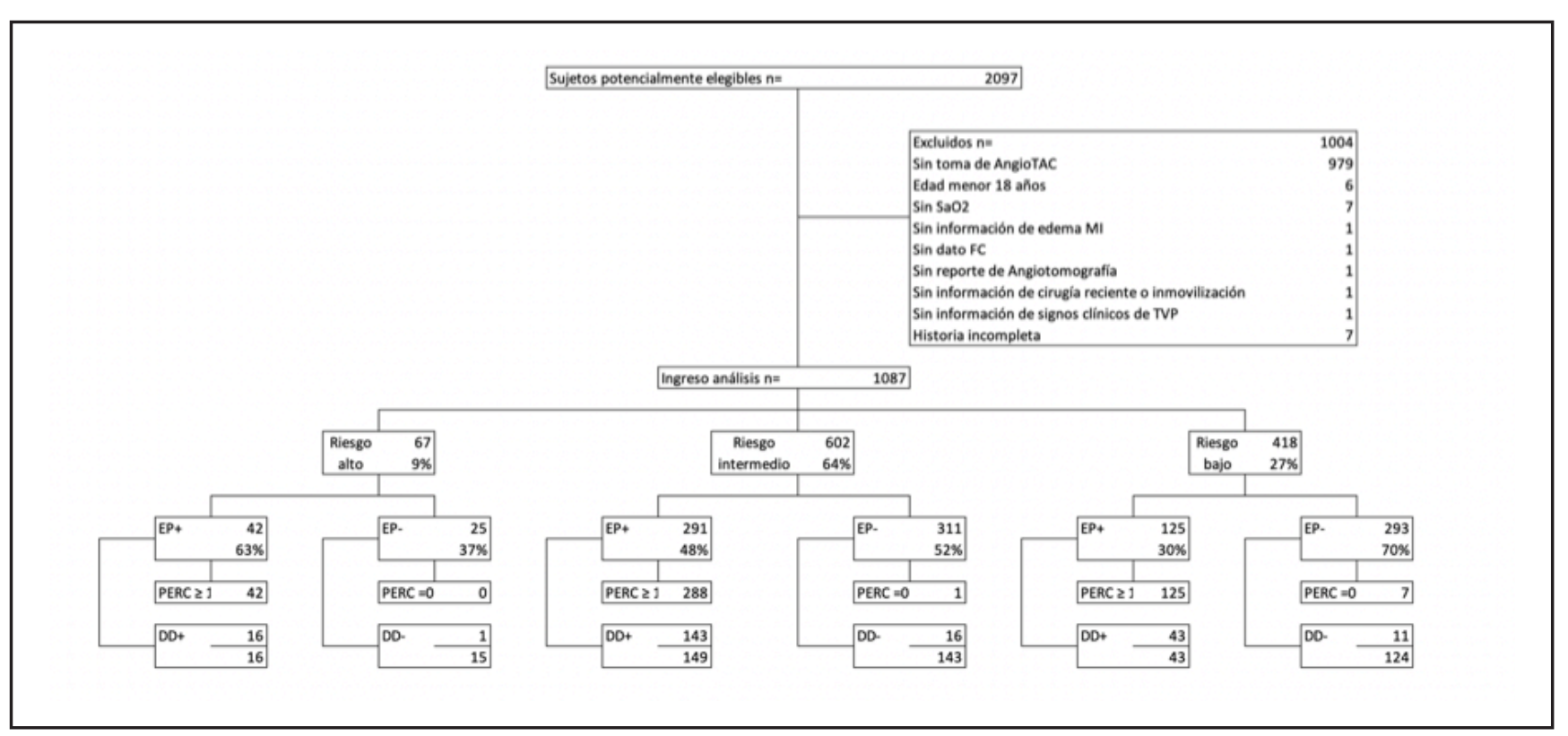

Figura 1. Flujograma de ingreso de pacientes al estudio. FC: frecuencia cardíaca, TVP: trombosis venosa profunda, angioTAC: angio-tomografía computarizada de arterias pulmonares, MI: miembro inferior, $\mathrm{SaO2:} \mathrm{saturación} \mathrm{de} \mathrm{oxígeno.}$ 


\section{Resultados}

Se ingresaron al análisis final un total de 1087 pacientes. En la Figura 1 se muestra el flujo de ingreso de pacientes al estudio. La EP se encontró en $42 \%$ de los sujetos evaluados. En la Tabla 1 se presentan las características sociodemográficas y antecedentes de la población en relación con la EP, donde se encontró una relación estadísticamente significativa para EP con demencia $(p=0.027)$, cáncer activo en el último año $(\mathrm{p}=0.022)$, antecedente de TEP $(\mathrm{p}=0.035)$ y el uso de anticonceptivos orales en mujeres $(p=0.016)$. En la Tabla 2 se presentan las características clínicas y hallazgos al examen físico con EP, se encontró una relación estadísticamente significativa con EP para dolor torácico $(\mathrm{p}=0.001)$, hemoptisis $(\mathrm{p}=0.035)$ y dolor en miembro inferior unilateral $(\mathrm{p}=0.010)$, junto con una

Tabla 1. Características generales de la población y el diagnóstico de embolia pulmonar.

\begin{tabular}{|c|c|c|c|c|}
\hline & $\begin{array}{l}\text { Población total } \\
\quad \mathrm{n}=1087\end{array}$ & $\mathrm{EP} n=458$ & $\operatorname{Sin} \mathrm{EP} n=629$ & Valor $\mathbf{p}$ \\
\hline Edad años x(de) & $59.8(19.3)$ & $61.3(19.7)$ & $58.7(18.8)$ & 0.028 \\
\hline Sexo femenino $\mathrm{n}(\%)$ & $585(53.8)$ & $242(52.8)$ & $343(54.5)$ & 0.58 \\
\hline \multicolumn{5}{|l|}{ Antecedentes n(\%) } \\
\hline Enfermedad cardiovascular & $260(23.9)$ & $73(15.9)$ & $187(29.7)$ & $<0.001$ \\
\hline IAM en los últimos tres meses & $25(2.3)$ & $8(1.7)$ & $17(2.7)$ & 0.299 \\
\hline Insuficiencia cardiaca congestiva & $69(6.3)$ & $28(6.1)$ & $41(6.5)$ & 0.787 \\
\hline Hipertensión arterial & $443(40.8)$ & $185(40.4)$ & $258(41)$ & 0.836 \\
\hline Fibrilación auricular & $58(5.3)$ & $26(5.7)$ & $32(5.1)$ & 0.669 \\
\hline Enfermedad vascular periferica & $54(5)$ & $28(2.6)$ & $26(2.4)$ & 0.138 \\
\hline Enfermedad cerebrovascular & $36(3.3)$ & $10(2.2)$ & $26(.1)$ & 0.076 \\
\hline Demencia & $29(2.7)$ & $18(3.9)$ & $11(1.7)$ & 0.028 \\
\hline Valvulopatía & $23(2.1)$ & $5(1.1)$ & $18(2.9)$ & 0.045 \\
\hline EPOC & $183(16.8)$ & $71(15.5)$ & $112(17.8)$ & 0.316 \\
\hline Asma & $25(2.3)$ & $4(0.87)$ & $21(3.3)$ & 0.007 \\
\hline Fibrosis pulmonar & $15(1.4)$ & $4(0.87)$ & $11(1.7)$ & 0.222 \\
\hline Patología del tejido conectivo & $32(2.9)$ & $7(1.5)$ & $25(4)$ & 0,018 \\
\hline Enfermedades autoinmunes & $69(6.3)$ & $27(5.9)$ & $42(6.7)$ & 0.602 \\
\hline Trastorno de la coagulación & $41(3.8)$ & $17(3.7)$ & $24(3.8)$ & 0.929 \\
\hline Diabetes mellitus & $130(12)$ & $53(11.6)$ & $77(12.2)$ & 0.737 \\
\hline Hemiplejía & $8(0.7)$ & $4(0.87)$ & $4(0.64)$ & 0.651 \\
\hline Obesidad (IMC > 30) & $53(4.9)$ & $25(5.5)$ & $28(4.5)$ & 0.447 \\
\hline Inmovilización por más de tres días & $182(16.7)$ & $89(19.4)$ & $93(14.8)$ & 0.043 \\
\hline Cirugía en las últimas cuatro semanas & $236(21.7)$ & $101(22.1)$ & $135(21.5)$ & 0.816 \\
\hline Reemplazo de cadera o rodilla & $42(3.9)$ & $15(3.3)$ & $27(4.3)$ & 0.39 \\
\hline Lesión de la médula espinal & $6(0.6)$ & $2(0.44)$ & $4(0.64)$ & 0.662 \\
\hline Trauma en las últimas cuatro semanas & $116(10.7)$ & $42(9.2)$ & $74(11.8)$ & 0.171 \\
\hline Fractura de MI en las últimas cuatro semanas & $75(6.9)$ & $30(6.6)$ & $45(7.2)$ & 0.698 \\
\hline Antecedente de malignidad & $132(12.1)$ & $63(13.8)$ & $69(11)$ & 0.165 \\
\hline Cáncer activo en el último año & $77(7.1)$ & $42(9.2)$ & $35(5.6)$ & 0.022 \\
\hline Uso de hormonas orales & $12(1.1)$ & $9(2)$ & $3(0.48)$ & 0.015 \\
\hline Antecedente de TVP & $109(10)$ & $49(10.7)$ & $60(9.5)$ & 0.53 \\
\hline Antecedente de TEP & $64(5.9)$ & $35(7.6)$ & $29(4.6)$ & 0.036 \\
\hline VIH/SIDA & $3(0.3)$ & $1(0.22)$ & $2(0.32)$ & 0.757 \\
\hline
\end{tabular}


TRABAJOS ORIGINALES • PERC como predictor de embolia pulmonar a gran altitud

Tabla 2. Signos y síntomas de la población y el diagnóstico de embolia pulmonar.

\begin{tabular}{|c|c|c|c|c|}
\hline & Población total n=1087 & $\mathrm{EP} n=458$ & Sin $E P n=629$ & Valor $\mathbf{p}$ \\
\hline \multicolumn{5}{|l|}{ Sintomatología n(\%) } \\
\hline Disnea aguda & $705(64.9)$ & $289(63.1)$ & $416(66.1)$ & 0.301 \\
\hline Dolor torácico & $581(53.4)$ & $271(59.2)$ & $310(49.3)$ & 0.001 \\
\hline Hemoptisis & $66(6.1)$ & $36(7.9)$ & $30(4.8)$ & 0.035 \\
\hline Dolor en MI unilateral & $142(13.1)$ & $74(16.2)$ & $68(10.8)$ & 0.01 \\
\hline \multicolumn{5}{|l|}{ Examen físico n(\%) } \\
\hline Edema unilateral de MI & $140(12.9)$ & $74(16.2)$ & $66(10.5)$ & 0.006 \\
\hline Dolor a la palpación & $119(10.9)$ & $66(14.4)$ & $53(8.4)$ & 0.002 \\
\hline Signos de TVP & $111(10.9)$ & $65(14.2)$ & $46(7.3)$ & $<0.001$ \\
\hline Venas colaterales no varicosas & $10(0.9)$ & $4(0.9)$ & $6(0.95)$ & 0.891 \\
\hline Distensión venosa de la extremidad afectada & $11(1)$ & $6(1.3)$ & $5(0.79)$ & 0.402 \\
\hline Diferencia de diámetro de $\operatorname{los} \mathrm{MI}>3 \mathrm{~cm}$ & $34(3.1)$ & $23(5)$ & $11(1.7)$ & 0.002 \\
\hline \multicolumn{5}{|l|}{ Signos vitales $\mathrm{x}(\mathrm{de})$} \\
\hline Temperatura & $36,5(1.2)$ & $36.4(1.7)$ & $36.5(0.3)$ & 0.159 \\
\hline Frecuencia cardiaca & $91(19.1)$ & $93(18.5)$ & $89.6(19.5)$ & 0.003 \\
\hline Presión arterial sistólica & $124(21.3)$ & $124.2(21.2)$ & $123.9(21.4)$ & 0.972 \\
\hline Presión arterial diastólica & $73.8(14)$ & $74.6(13.4)$ & $73.2(14.4)$ & 0.101 \\
\hline Saturación de O2 (SaO2) & $89.1(7.1)$ & $88.9(7.3)$ & $89.3(6.9)$ & 0.91 \\
\hline Fracción inspirada de $\mathrm{O} 2$ (FiO2) & $24.6(9)$ & $24.6(10.6)$ & $24.6(7.7)$ & 0.953 \\
\hline Relación $\mathrm{SaO} 2 / \mathrm{FIO} 2$ & $383.5(72.7)$ & $385.8(73.6)$ & $381.7(72)$ & 0.909 \\
\hline
\end{tabular}

relación estadísticamente significativa para la presencia de signos de TVP excluyendo la distensión venosa y la presencia de venas colaterales no varicosas. En el análisis de los signos vitales solo se encontró una relación estadísticamente significativa de EP con la frecuencia cardiaca $(\mathrm{P}=0.003)$.

En relación con los exámenes de laboratorio tomados a los pacientes, hay relación estadísticamente significativa con el diagnóstico de EP y la sobrecarga del ventrículo derecho evidenciada en electrocardiograma, los hallazgos en la radiografía de tórax de oligohemia, amputación de la arteria hiliar y presencia de consolidación debida a infarto pulmonar, los cuales presentaron un valor $\mathrm{p}<0.001$. Además se observó que los pacientes con diagnóstico de EP presentan valores mayores de dímero D con una mediana de $3.43 \mu \mathrm{g} / \mathrm{mL}$ (RIQ: 4.7) vs $1.31 \mu \mathrm{g} / \mathrm{mL}$ (RIQ: 1.53 ) como se observa en la Tabla 3.

En la Tabla 4 se presentan los resultados en relación de las diferentes variables utilizadas para el cálculo del puntaje de PERC y su relación con EP, además se muestra la relación con EP del puntaje PERC calculado utilizando la $\mathrm{SatO} 2<95 \%$ y SatO2<90\% y el diagnóstico de EP, donde un PERC $\geq 1$ calculado con Sat $<90 \%$ mostró una relación estadísticamente significativa con la presencia de EP $(\mathrm{p}=0.006)$. En la Tabla 5 se muestran los resultados de sensibilidad, especificidad y ACOR del puntaje PERC calculado con $\mathrm{SatO} 2<95 \%$ y $\mathrm{SatO} 2<90 \%$ en los pacientes clasificados en los diferentes grupos de riesgo por los puntajes de Wells, Ginebra y Pisa. Se encontró que el puntaje de PERC cuando se utiliza en su cálculo una SatO2 $<95 \%$ la sensibilidad fue de $99.3 \%$, especificidad de $1.3 \%$, falsos negativos de $0 \%$ y ACOR de 0.56 (IC95\%:0-52-0.59) (P=0.001) y cuando el puntaje PERC se calcula utilizando una $\mathrm{SatO} 2<90 \%$ la sensibilidad fue de $98.0 \%$, especificidad de $5.2 \%$, falsos negativos de $2.4 \%$ y ACOR de 0.60 (IC-95\%:0.54-0.66) ( $\mathrm{p}=0.002)$.

\section{Discusión}

En el presente estudio se encuentra que el utilizar la saturación de oxígeno con un punto de corte del $90 \%$ para 
Alirio Rodrigo Bastidas-Goyes y cols.

Tabla 3. Resultados de laboratorio, imagenología y desenlaces con el diagnóstico de embolia pulmonar.

\begin{tabular}{|c|c|c|c|c|}
\hline & Población total n=1087 & $E P n=458$ & Sin EP $n=629$ & Valor $\mathbf{p}$ \\
\hline \multicolumn{5}{|l|}{ Paraclínicos n(\%) } \\
\hline Toma de electrocardiograma & $714(65.7)$ & $293(64)$ & $421(66.9)$ & 0.31 \\
\hline Sobrecarga de VD & $99(9.1)$ & $60(13.1)$ & $39(6.2)$ & $<0.001$ \\
\hline Toma de radiografía de tórax & $876(80.6)$ & $341(7.5)$ & $535(85.1)$ & $<0.001$ \\
\hline Oligohemia & $11(1)$ & $8(1.7)$ & $3(0.5)$ & $<0.001$ \\
\hline Amputación de arteria hiliar & $4(0.4)$ & $4(0.9)$ & $0(0)$ & $<0.001$ \\
\hline Consolidación por infarto pulmonar & $33(3)$ & $23(5)$ & $10(1.6)$ & $<0.001$ \\
\hline Consolidación sin infarto pulmonar & $136(12.5)$ & $50(10.9)$ & $86(13.7)$ & $<0.001$ \\
\hline Edema pulmonar & $41(3.8)$ & $20(4.4)$ & $21(3.3)$ & $<0.001$ \\
\hline Dímero D M(RIQ) & $2.35(3.89)$ & $3.43(4.7)$ & $1.31(1.53)$ & $<0.001$ \\
\hline \multicolumn{5}{|l|}{ Trombosis de MI n(\%) } \\
\hline TVS & $32(2.9)$ & $21(4.6)$ & $11(1.7)$ & $<0.001$ \\
\hline TVP & $132(12.1)$ & $91(19.9)$ & $41(6.5)$ & $<0.001$ \\
\hline \multicolumn{5}{|l|}{ Desenlaces n (\%) } \\
\hline Ventilación mecánica & $112(10.3)$ & $44(9.6)$ & $68(10.8)$ & 0.519 \\
\hline Ingreso UCI & $284(26.1)$ & $132(28.8)$ & $152(24.2)$ & 0.084 \\
\hline
\end{tabular}

el cálculo de PERC, mantiene una alta sensibilidad y mejora la especificidad cuando se compara con un puntaje de PERC calculado con un punto de corte de $\mathrm{SatO} 2$ del $95 \%$ a gran altitud (2640 $\mathrm{msnm})$; estos resultados guardan relación con la disminución de la saturación de oxígeno que se presenta cuando aumenta la altitud y a los procesos de adaptación fisiológicos que se ven en sujetos que viven a alturas mayores de $2500 \mathrm{msnm}$. Nuestros hallazgos son similares a los reportados en estudios donde se aplicó el PERC a altitudes de $1500 \mathrm{msnm}$, donde se encontró que la utilización de la saturación de $90 \%$ para construir el puntaje mantenía una sensibilidad de 94.8-100\% y una especificidad de 17.8-

Tabla 4. Comparación de las variables del puntaje PERC y el diagnóstico de embolia pulmonar.

\begin{tabular}{|c|c|c|c|c|}
\hline & $\begin{array}{c}\text { Población total } \\
\text { n=1087 }\end{array}$ & $\mathrm{EP} n=458$ & Sin EP $n=629$ & Valor $\mathbf{p}$ \\
\hline PERC edad $>50$ años $n(\%)$ & $761(70)$ & $328(71.6)$ & $433(68.8)$ & 0.324 \\
\hline PERC FC >100 n(\%) & $381(35.1)$ & $176(38.4)$ & 205 (32.6) & 0.046 \\
\hline PERC $\mathrm{SaO} 2<95 \%$ al ambiente $\mathrm{n}(\%)$ & $979(90.1)$ & $406(88.6)$ & $573(91.1)$ & 0.182 \\
\hline PERC SaO $2<90 \%$ al ambiente $\mathrm{n}(\%)$ & $643(59.2)$ & $267(58.3)$ & $376(59.8)$ & 0.624 \\
\hline PERC hemoptisis $\mathrm{n}(\%)$ & $66(6.1)$ & $36(7.9)$ & $30(4.8)$ & 0.035 \\
\hline PERC uso estrógenos n(\%) & $12(1.1)$ & $9(0.8)$ & $3(0.3)$ & 0.02 \\
\hline PERC antecedente TVP o TEP n $(\%)$ & $149(13.7)$ & $71(15.5)$ & $78(12.4)$ & 0.142 \\
\hline PERC antecedente de trauma o cirugia $n(\%)$ & $282(25.9)$ & $117(25.5)$ & $165(26.2)$ & 0.799 \\
\hline PERC edema unilateral MI n(\%) & $140(12.9)$ & $74(16.2)$ & $66(10.5)$ & 0.006 \\
\hline PERC SatO2<95\% $\geq 1 \mathrm{n}(\%)$ & $1076(99)$ & $455(99.3)$ & $621(98.7)$ & 0.316 \\
\hline PERC SatO2<90\% $\geq 1 \mathrm{n}(\%)$ & $1045(96.1)$ & $449(98)$ & $596(94.8)$ & 0.006 \\
\hline
\end{tabular}


TRABAJOS ORIGINALES • PERC como predictor de embolia pulmonar a gran altitud

Tabla 5. Desempeño del PERC con SatO2<95\% y PERC con Sat<90\% para embolia pulmonar categorizado por puntajes de Wells. Ginebra y Pisa.

\begin{tabular}{|c|c|c|c|c|}
\hline & Sensibilidad & Especificidad & ACOR (IC-95\%) & Valor $\mathbf{p}$ \\
\hline PERC SatO2<95\% & 99.3 & 1.3 & $0.56(0.52-0.59)$ & 0.001 \\
\hline \multicolumn{5}{|l|}{ Wells dicotómico } \\
\hline No probable & 99.1 & 1.8 & $0.53(0.48-0.57)$ & 0.223 \\
\hline Probable & 99.6 & 0.0 & $0.49(0.44-0.55)$ & 0.801 \\
\hline \multicolumn{5}{|l|}{ Wells tres niveles } \\
\hline Riesgo bajo & 100.0 & 2.4 & $0.56(0.50-0.62)$ & 0.049 \\
\hline Riesgo intermedio & 99.0 & 0.3 & $0.48(0.44-0.53)$ & 0.482 \\
\hline Riesgo alto & 100.0 & 0.0 & $0.52(0.37-0.67)$ & 0.775 \\
\hline \multicolumn{5}{|l|}{ Ginebra } \\
\hline Riesgo bajo & 98.8 & 3.5 & $0.53(0.45-0.60)$ & 0.459 \\
\hline Riesgo intermedio & 99.4 & 3.5 & $0.51(0.46-0.55)$ & 0.905 \\
\hline Riesgo alto & 100.0 & 0.0 & $0.39(0.27-0.51)$ & 0.081 \\
\hline \multicolumn{5}{|l|}{ Pisa } \\
\hline$\leq 10 \%$ & 97.8 & 0.8 & $0.54(0.44-0.64)$ & 0.422 \\
\hline$>10 \% \leq 50 \%$ & 100.0 & 1.3 & $0.55(0.49-0.61)$ & 0.093 \\
\hline$>50 \% \leq 90 \%$ & 100.0 & 3.4 & $0.5(0.36-0.64)$ & 0.971 \\
\hline$>90 \%$ & 100.0 & 0.0 & $0.35(0.0-0.71)$ & 0.497 \\
\hline PERC SatO2<90\% & 98.0 & 5.2 & $0.55(0.52-0.59)$ & 0.003 \\
\hline \multicolumn{5}{|l|}{ Wells dicotómico } \\
\hline No probable & 96.6 & 7.6 & $0.55(0.50-0.59)$ & 0.040 \\
\hline Probable & 99.6 & 0.0 & $0.47(0.41-0.42)$ & 0.251 \\
\hline \multicolumn{5}{|l|}{ Wells tres niveles } \\
\hline Riesgo bajo & 97.6 & 7.8 & $0.6(0.54-0.65)$ & 0.002 \\
\hline Riesgo intermedio & 97.9 & 3.2 & $0.47(0.42-0.51)$ & 0.140 \\
\hline Riesgo alto & 100.0 & 0.0 & $0.49(0.34-0.64)$ & 0.881 \\
\hline \multicolumn{5}{|l|}{ Ginebra } \\
\hline Riesgo bajo & 92.7 & 13.1 & $0.55(0.47-0.62)$ & 0.218 \\
\hline Riesgo intermedio & 99.1 & 1.8 & $0.5(0.45-0.54)$ & 0.905 \\
\hline Riesgo alto & 100.0 & 0.0 & $0.41(0.29-0.52)$ & 0.145 \\
\hline \multicolumn{5}{|l|}{ Pisa } \\
\hline$\leq 10 \%$ & 95.7 & 5.6 & $0.56(0.46-0.66)$ & 0.236 \\
\hline$>10 \% \leq 50 \%$ & 99.3 & 4.9 & $0.55(0.49-0.60)$ & 0.139 \\
\hline$>50 \% \leq 90 \%$ & 100.0 & 3.4 & $0.55(0.41-0.69)$ & 0.471 \\
\hline$>90 \%$ & 100.0 & 0.0 & $0.29(0.0-0.63)$ & 0.350 \\
\hline
\end{tabular}


$22 \%(25,26)$, además, este puntaje mantiene un poder de discriminación para el diagnóstico de EP en pacientes clasificados de bajo riesgo por el puntaje de Wells en esta población de estudio $(13,25,26)$.

Los valores de oxigenación disminuyen con el aumento de la altitud, la disminución de la presión atmosférica lleva a una disminución de la presión alveolar de oxígeno y del intercambio gaseoso que puede finalmente disminuir los valores de la $\mathrm{SatO} 2$ en sujetos que viven a grandes alturas (14). De este modo, un valor de $\mathrm{SatO} 2<95 \%$ a altitudes superiores de 2500 m.s.n.m no necesariamente estaría relacionado con la presencia de EP. En un estudio realizado en Bogotá (24), valores entre 90 y 95\% se encuentran en el rango de normalidad de la mayoría de la población. Asignar un punto a los valores de la $\mathrm{SatO} 2<95 \%$ para el cálculo de PERC aumenta la sensibilidad del puntaje, pero al compararse ésta con la obtenida cuando se calcula el PERC asignado un punto a la $\mathrm{SatO} 2<90 \%$ en la población de bajo riesgo a gran altitud la sensibilidad obtenida no varía más de dos puntos porcentuales y se mantiene por encima de $97 \%$, en este mismo sentido, la proporción de falsos negativos con el PERC calculado con SatO2 $<90 \%$ fue de $2.4 \%$ porcentaje menor a lo reportado en la literatura donde los falsos negativos pueden encontrarse entre el 5.4-6.4\% $(13,27,28)$. La presencia de falsos negativos utilizando dímero $\mathrm{D}$ o puntajes como el PERC podrían ser el resultado de la evaluación de pacientes con pequeñas embolias subsegmentarias que generan cuadros poco sintomáticos y que generalmente tienen un curso benigno aún sin tratamiento $(22,29)$.

Otras variables que pueden influir en los valores de saturación son la edad y la presencia de comorbilidades, nuestra población tiene un promedio de edad 12 años mayor al estudio de Kline et al (12), donde se realizaron las validaciones originales del PERC, sin embargo, esta variación de edad en diferentes poblaciones $(12,13,22)$, no parece influir de manera importante en el desempeño del puntaje, en cuanto a la presencia de comorbilidades en nuestro estudio hay una mayor proporción de sujetos con antecedente de enfermedad pulmonar cuando se compara con otros estudios de validación del PERC (12), sin embargo, la proporción de sujetos con enfermedad pulmonar obstructiva crónica en nuestra población tiene una distribución similar entre los sujetos con y sin EP, y los sujetos con ASMA y fibrosis pulmonar son sólo del 5\%, situación que creemos difícilmente podría influir en los resultados obtenidos del poder discriminatorio del PERC utilizando una Sat $<90 \%$. Por otra parte, se encontró que la demencia se encontraba en relación con EP, situación que puede ser explicada a una disminución de la movilidad y presencia de comorbilidades en estos pacientes, esta condición poco referida en estudios previos se ha relacionado con mayor severidad y aumento de la mortalidad en los pacientes con EP (30).

$\mathrm{Al}$ realizar el análisis del desempeño del PERC calculado con $\mathrm{SatO} 2<95 \%$ y el PERC calculado con $\mathrm{SatO}<<0 \%$ en los pacientes clasificados de bajo riesgo por las escalas de Wells,
Ginebra y Pisa, encontramos un mejor poder de clasificación del puntaje PERC cuando los pacientes son clasificados con riesgo bajo mediante la escala de Wells $(<2)$ y Wells simplificado $(<4)$, situación similar a la reportada en la literatura. Madsen et al. utiliza la clasificación de Wells simplificado $(<4)$, y Wolf et al, utiliza la clasificación de Wells $(<2)$ en la evaluación del PERC obteniendo un buen rendimiento de este puntaje para descartar EP $(25,26)$. Por el contrario, cuando se evalúa el rendimiento del PERC, en pacientes de bajo riesgo clasificados por la escala de Ginebra vs una clasificación de bajo riesgo por criterio clínico (bajo riesgo definido como pacientes con un riesgo lo suficientemente bajo como para ser descartado mediante la toma de dímero D) se observa que el rendimiento del puntaje PERC disminuye cuando los pacientes fueron clasificados de bajo riesgo con el puntaje de Ginebra (13). Por lo tanto, con los datos obtenidos se sugiere que la clasificación inicial de riesgo para EP se realice a través de la escala de Wells y de esta manera conseguir un mejor desempeño en la utilización del PERC.

En cuanto a las debilidades, debido a que el presente estudio es de carácter retrospectivo, la calidad de la información está sujeta a lo consignado en la historia clínica y a la capacidad de los miembros del grupo de investigación de recolectar datos adecuados, sin embargo, el equipo de investigación tiene el conocimiento médico suficiente para una adecuada interpretación de la información clínica. Al ser un estudio desarrollado en un hospital de alta complejidad, existe el riesgo de sesgo de selección y espectro de la enfermedad, donde la proporción de sujetos con riesgo intermedio y alto puede ser mayor a la proporción de sujetos con riesgo bajo, los sujetos con riesgo bajo no son llevados a la realización de ATC de arterias pulmonares de manera rutinaria y por esta razón llegar a ser excluidos del análisis final y los sujetos con riesgo alto pueden presentar complicaciones severas como muerte temprana o imposibilidad de evaluación con ATC por inestabilidad hemodinámica; esta situación, podría afectar y aumentar la frecuencia de EP en la población estudiada, sensibilidad y falsos negativos, no obstante, el estudio cuenta con un tamaño de muestra suficiente para poder llegar a conclusiones válidas. Nuestros resultados plantean como posibles estudios futuros, la evaluación económica del uso de estrategias basadas en estas reglas de predicción clínica en comparación o en conjunto con el dímero $\mathrm{D}$ en población colombiana.

\section{Conclusión}

El puntaje PERC calculado con una saturación de oxígeno $<90 \%$ tiene un rendimiento diagnóstico similar al puntaje PERC calculado con una saturación de oxígeno de $<95 \%$ para descartar EP en pacientes clasificados en bajo riesgo a gran altitud (>2500 msnm). La capacidad para descartar EP con el puntaje PERC se mantiene estadísticamente significativa con la clasificación de bajo riesgo por escala de Wells y pierde significancia estadística al utilizar la clasificación de bajo riesgo con los puntajes de Ginebra y Pisa. El puntaje 
PERC puede ser tenido en cuenta como una herramienta diagnóstica adicional para la evaluación de pacientes con riesgo de EP a gran altitud. Estos resultados no pueden ser extrapolables a poblaciones especiales como lo son mujeres embarazadas y menores de 18 años.

\section{Referencias}

1. Dennis R, Rodríguez MN. Estudio nacional sobre tromboembolismo venoso en población hospitalaria en Colombia. Acta Med Colomb. 1996;21:55-63.

2. Friedman T, Winokur RS, Quencer KB, Madoff DC. Patient Assessment: Clinical Presentation, Imaging Diagnosis, Risk Stratification, and the Role of Pulmonary Embolism Response Team. Semin Intervent Radiol. 2018;35(2):116-21.

3. Stein PD, Beemath A, Matta F, Weg JG, Yusen RD, Hales CA, et al. Clinical characteristics of patients with acute pulmonary embolism: data from PIOPED II. Am J Med. 2007;120(10):871-9.

4. Yap KS, Kalff V, Turlakow A, Kelly MJ. A prospective reassessment of the utility of the Wells score in identifying pulmonary embolism. Medical journal of Australia. 2007;187(6):333-6.

5. van Belle A, Buller HR, Huisman MV, Huisman PM, Kaasjager K, Kamphuisen PW, et al. Effectiveness of managing suspected pulmonary embolism using an algorithm combining clinical probability, D-dimer testing, and computed tomography. Jama. 2006;295(2):172-9.

6. Yap KS, Kalff V,Turlakow A, Kelly MJ.A prospective reassessment of the utility of the Wells score in identifying pulmonary embolism. Med J Aust. 2007;187(6):333-6.

7. Miniati M, Monti S, Bottai M. A structured clinical model for predicting the probability of pulmonary embolism. Am J Med. 2003;114(3):173-9.

8. Le Gal G, Righini M, Roy PM, Sanchez O, Aujesky D, Bounameaux H, et al. Prediction of pulmonary embolism in the emergency department: the revised Geneva score. Ann Intern Med. 2006;144(3):165-71.

9. Bass AR, Fields KG, Goto R, Turissini G, Dey S, Russell LA. Clinical Decision Rules for Pulmonary Embolism in Hospitalized Patients: A Systematic Literature Review and Meta-analysis. Thromb Haemost. 2017;117(11):2176-85.

10. BAstidAs-goyes Ar, FAizAl-gómez Ni, ortiz-ramírez S, aguirre-ContreraS G. Rendimiento diagnóstico de tres reglas de predicción clínica para embolia pulmonar. Act Med Colombia. 2020;45.

11. Miniati M, Bottai M, Monti S. Comparison of 3 clinical models for predicting the probability of pulmonary embolism. Medicine (Baltimore). 2005;84(2):107-14.

12. Kline J, Mitchell A, Kabrhel C, Richman P, Courtney D. Clinical criteria to prevent unnecessary diagnostic testing in emergency department patients with suspected pulmonary embolism. Journal of Thrombosis and Haemostasis. 2004;2(8):1247-55.

13. Penaloza A, Verschuren F, Dambrine S, Zech F, Thys F, Roy P-M. Performance of the Pulmonary Embolism Rule-out Criteria (the PERC rule) combined with low clinical probability in high prevalence population. Thrombosis research. 2012;129(5):e189-e93.

14. Villacorta-Cordova F, Carrillo Coba E, Zubia-Olaskoaga F, Tinoco-Solórzano A. Comparación de los valores normales de gases arteriales entre la altitud y el nivel del mar del Ecuador. Revista de Medicina Intensiva y Cuidados Críticos. 2020;13:88-91.
15. Gonzalez-Garcia M, Dario Maldonado D, Barrero M, Casas A, Perez-Padilla $\mathbf{R}$, Torres-Duque C. Arterial blood gases and ventilation at rest by age and sex in an adult Andean population resident at high altitude. Eur J Appl Physiol. 2020:2729-36.

16. Lucassen W, Geersing GJ, Erkens PM, Reitsma JB, Moons KG, Buller H, et al. Clinical decision rules for excluding pulmonary embolism: a meta-analysis. Ann Intern Med. 2011;155(7):448-60.

17. Ceriani E, Combescure C, Le Gal G, Nendaz M, Perneger T, Bounameaux H, et al. Clinical prediction rules for pulmonary embolism: a systematic review and meta-analysis. J Thromb Haemost. 2010;8(5):957-70.

18. Wells PS, Ginsberg JS, Anderson DR, Kearon C, Gent M, Turpie AG, et al. Use of a clinical model for safe management of patients with suspected pulmonary embolism. Ann Intern Med. 1998;129(12):997-1005.

19. Schouten HJ, Geersing GJ, Oudega R, van Delden JJ, Moons KG, Koek HL. Accuracy of the Wells clinical prediction rule for pulmonary embolism in older ambulatory adults. J Am Geriatr Soc. 2014;62(11):2136-41.

20. Klok FA, Mos IC, Nijkeuter M, Righini M, Perrier A, Le Gal G, et al. Simplification of the revised Geneva score for assessing clinical probability of pulmonary embolism. Arch Intern Med. 2008;168(19):2131-6.

21. Cronin P, Dwamena BA.A Clinically Meaningful Interpretation of the Prospective Investigation of Pulmonary Embolism Diagnosis (PIOPED) Scintigraphic Data. Acad Radiol. 2017;24(5):550-62.

22. Freund Y, Cachanado M, Aubry A, Orsini C, Raynal PA, Feral-Pierssens AL, et al. Effect of the Pulmonary Embolism Rule-Out Criteria on Subsequent Thromboembolic Events Among Low-Risk Emergency Department Patients: The PROPER Randomized Clinical Trial. Jama. 2018;319(6):559-66.

23. Fabia Valls MJ, van der Hulle T, den Exter PL, Mos IC, Huisman MV, Klok FA. Performance of a diagnostic algorithm based on a prediction rule, D-dimer and CTscan for pulmonary embolism in patients with previous venous thromboembolism. A systematic review and meta-analysis. Thromb Haemost. 2015;113(2):406-13.

24. Gonzalez-Garcia M BM, Casas A, Torres-Duque CA, Maldonado D. Reference values for arterial blood gases at an altitude of 2640 meters. Am J Respir Crit Care Med. 2013;187:A4852.

25. Madsen T, Jedick R, Teeples T, Carlson M, Steenblik J. Impact of altitudeadjusted hypoxia on the Pulmonary Embolism Rule-out Criteria. American Journal of Emergency Medicine. 2018;37:281-5.

26. Wolf SJ, McCubbin TR, Nordenholz KE, Naviaux NW, Haukoos JS. Assessment of the pulmonary embolism rule-out criteria rule for evaluation of suspected pulmonary embolism in the emergency department. American Journal of Emergency Medicine. 2008;226(181-185).

27. Righini M, Le Gal G, Perrier A, Bounameaux H. More on: clinical criteria to prevent unnecessary diagnostic testing in emergency department patients with suspected pulmonary embolism. J Thromb Haemost. 2005;3:188-9.

28. HUGLI O, RIGHINI M, GAL GL, ROY M, SANCHEZ O, VERSCHUREN F, et al. The pulmonary embolism rule-out criteria (PERC) rule does not safely exclude pulmonary embolism. Journal of Thrombosis and Haemostasis. 2010;9:300-4.

29. Wahl WL, Ahrns KS, Zajkowski PJ, Brandt M-M, Proctor M, Arbabi S, et al. Normal d-dimer levels do not exclude thrombotic complications in trauma patients. Surgery. 2003;134(4):529-32.

30. Nuñez MJ, Villalba JC, Cebrián E, Visoná A, Lopez-Jimenez L, Núñez M, et al. Venous thromboembolism in immobilized patients with dementia. Findings from the RIETE registry. Thrombosis Research. 2012;130:173-7. 